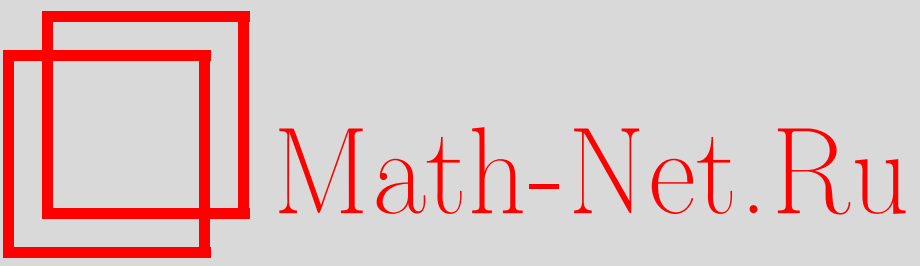

Ю. Н. Субботин, С. А. Теляковский, Относительные поперечники классов дифференцируемых функций в метрике $L^{2}$, УMH, 2001, том 56, выпуск 4, 159-160

DOI: https://doi.org/10.4213/rm432

Использование Общероссийского математического портала Math-Net.Ru подразумевает, что вы прочитали и согласны с пользовательским соглашением

http://www. mathnet.ru/rus/agreement

Параметры загрузки:

IP: 3.89 .185 .249

26 апреля 2023 г., 10:14:20 


\title{
ОТНОСИТЕЛЬНЫЕ ПОПЕРЕЧНИКИ КЛАССОВ ДИФФЕРЕНЦИРУЕМЫХ ФУНКЦИЙ В МЕТРИКЕ $L^{2}$
}

\author{
Ю. Н. СуББотин, С. А. ТЕляковский
}

Понятие относительного поперечника введено В.Н. Коноваловым [1]. Пусть $W$ и $V$ центрально-симметричные множества банахова пространства $X$. Тогда $n$-мерным поперечником множества $W$ относительно множества $V$ в пространстве $X$ назьвается величина

$$
K_{n}(W, V, X):=\inf _{l_{n}} \sup _{f \in W} \inf _{g \in V \cap l_{n}}\|f-g\|_{X},
$$

где нижняя грань берется по всем $n$-мерным подпространствам $l_{n}$ пространства $X$.

При $V=X$ относительньй поперечник $K_{n}(W, X, X)$ совпадает с $n$-мерным колмогоровским поперечником множества $W$ в пространстве $X$, который будем обозначать $d_{n}(W, X)$. Понятно, что для любого множества $V$ справедливо неравенство

$$
K_{n}(W, V, X) \geqslant d_{n}(W, X) .
$$

Сейчас нас будет интересовать вопрос, когда в (1) имеет место знак равенства.

Пусть $M W_{C}^{r}, r=1,2, \ldots,-$ класс $2 \pi$-периодических функций, у которых производная порядка $r-1$ удовлетворяет условию Липшица $\left|f^{(r-1)}\left(x^{\prime}\right)-f^{(r-1)}\left(x^{\prime \prime}\right)\right| \leqslant M\left|x^{\prime}-x^{\prime \prime}\right|$, а $M W_{L}^{r}$, $r=1,2 \ldots,-$ класс $2 \pi$-периодических функций, у которых вариация производной порядка $r-1$ на периоде не превосходит числа $M$. При $M=1$ пишем $W_{C}^{r}$ и $W_{L}^{r}$ соответственно.

В [2] приведен обзор основных резултатов об относительных поперечниках

$$
K_{n}\left(W_{C}^{r}, M W_{C}^{r}, C\right) \text { и } K_{n}\left(W_{L}^{r}, M W_{L}^{r}, L\right),
$$

которьй мы не будем здесь повторять. Отметим только, что из результатов В.Н. Коновалова и В. $\Phi$. Бабенко следует, что при $M=1$ и $r \geqslant 3$ указанные относительные поперечники при $n \rightarrow \infty$ имеют порядок $n^{-2}$, а при фиксированном $M>1$ они для всех $r$ имеют тот же порядок, что и соответствующие колмогоровские поперечники.

В [2] найдены условия на $M$, достаточные, для того чтобы имели место равенства

$$
K_{n}\left(W_{C}^{r}, M W_{C}^{r}, C\right)=d_{n}\left(W_{C}^{r}, C\right) \text { и } K_{n}\left(W_{L}^{r}, M W_{L}^{r}, L\right)=d_{n}\left(W_{L}^{r}, L\right) .
$$

В настоящей работе рассматривается аналогичная задача для относителных поперечников $K_{n}\left(W_{L^{2}}^{r}, M W_{L^{2}}^{r}, L^{2}\right)$, где $M W_{L^{2}}^{r}, r=1,2, \ldots,-$ класс функций периода $2 \pi$, для $L^{2}$-нормы производной порядка $r$ которых выполняется оценка

$$
\left(\frac{1}{\pi} \int_{-\pi}^{\pi}\left|f^{(r)}(x)\right|^{2} d x\right)^{1 / 2} \leqslant M
$$

Заметим, что при $M=0$ класс $M W_{L^{2}}^{r}$ представляет собой одномерное пространство констант.

В частности, будет установлено, что для $W_{L^{2}}^{r}$ в отличие от соответствующих результатов для классов $W_{C}^{r}$ и $W_{L}^{r}$ наименьшее значение $M$, при котором указанные относительные поперечники совпадают с колмогоровскими, меньше 1.

Известно (см. [3; $§ 10.3])$, что

$$
d_{2 m-1}\left(W_{L^{2}}^{r}, L^{2}\right)=d_{2 m}\left(W_{L^{2}}^{r}, L^{2}\right)=m^{-r}, \quad m=1,2, \ldots .
$$

Теорема. Пусть $m$ и $r$ - натуральные числа. Наименьшее число $M=M(m, r)$, при котором справедливы равенства

$$
K_{2 m-1}\left(W_{L^{2}}^{r}, M W_{L^{2}}^{r}, L^{2}\right)=K_{2 m}\left(W_{L^{2}}^{r}, M W_{L^{2}}^{r}, L^{2}\right)=m^{-r},
$$

равно

$$
1-m^{-r} .
$$

ДокАЗАТЕЛЬСтво. Так как функция $\cos x$ принадлежит классу $W_{L^{2}}^{r}$, то для произвольного числа $M$ имеем

$$
K_{2 m}\left(W_{L^{2}}^{r}, M W_{L^{2}}^{r}, L^{2}\right) \geqslant \inf _{g \in M W_{L^{2}}^{r}}\|\cos x-g(x)\|_{L^{2}}
$$

Работа выполнена при финансовой поддержке РФФИ - ГФЕН Китая (грант № 99-01-39006). 
Если $M<1$, то в силу ортогональности тригонометрической системы

$$
\inf _{g \in M W_{L^{2}}^{r}}\|\cos x-g(x)\|_{L^{2}}=1-M
$$

Поэтому из (2) вытекает оценка

$$
K_{2 m}\left(W_{L^{2}}^{r}, M W_{L^{2}}^{r}, L^{2}\right) \geqslant 1-M .
$$

Значит, для того чтобы выполнялось равенство

необходимо следующее условие на $M$

$$
K_{2 m}\left(W_{L^{2}}^{r}, M W_{L^{2}}^{r}, L^{2}\right)=m^{-r},
$$

т.е. условие

$$
1-M \leqslant m^{-r}
$$

$$
M \geqslant 1-m^{-r} .
$$

Покажем теперь, что при $M=1-m^{-r}$ справедлива оценка

Пусть функция $f(x) \in W_{L^{2}}^{r}$ и

$$
K_{2 m-1}\left(W_{L^{2}}^{r}, M W_{L^{2}}^{r}, L^{2}\right) \leqslant m^{-r} .
$$

$$
\frac{a_{0}}{2}+\sum_{k=1}^{\infty}\left(a_{k} \cos k x+b_{k} \sin k x\right)
$$

- ее ряд Фурье. Тогда

$$
\sum_{k=1}^{\infty} k^{2 r}\left(a_{k}^{2}+b_{k}^{2}\right) \leqslant 1
$$

Рассмотрим полином

$$
T_{m-1}(x)=\frac{a_{0}}{2}+\sum_{k=1}^{m-1}\left(1-m^{-r}\right)\left(a_{k} \cos k x+b_{k} \sin k x\right) .
$$

Так как

$$
\left\|T_{m-1}^{(r)}\right\|_{L^{2}}^{2}=\left(1-m^{-r}\right)^{2} \sum_{k=1}^{m-1} k^{2 r}\left(a_{k}^{2}+b_{k}^{2}\right)
$$

то из (4) следует, что

$$
T_{m-1} \in\left(1-m^{-r}\right) W_{L^{2}}^{r}
$$

Оценим приближение функции $f$ полиномом $T_{m-1}$ :

$$
\left\|f-T_{m-1}\right\|_{L^{2}}^{2}=m^{-2 r} \sum_{k=1}^{m-1}\left(a_{k}^{2}+b_{k}^{2}\right)+\sum_{k=m}^{\infty}\left(a_{k}^{2}+b_{k}^{2}\right) \leqslant m^{-2 r} \sum_{k=1}^{\infty} k^{2 r}\left(a_{k}^{2}+b_{k}^{2}\right) \leqslant m^{-2 r} .
$$

Итак, каждая функция из $W_{L^{2}}^{r}$ может быть приближена по норме пространства $L^{2}$ с погрешностью $m^{-r}$ тригонометрическими полиномами порядка $m-1$, принадлежащими классу $\left(1-m^{-r}\right) W_{L^{2}}^{r}$. А это означает справедливость неравенства (3) при $M=1-m^{-r}$.

Таким образом, теорема доказана.

\section{СПИСОК ЛИТЕРАТУРЫ}

[1] В. Н. Коновалов // Матем. заметки. 1984. Т. 35. С. 369-380. [2] Ю. Н. Субботин, С. А. Теляковский // Матем. заметки. 1999. Т. 65. С. 871-879. [3] Н. П. Корнейчук. Экстремальные задачи теории приближений. М.: Наука, 1976.

Институт математики и механики УрО РАН;

Математический институт им. В. А. Стеклова РАН
Принято редколлегией 21.06 .2001 